\title{
Arqueomagnetismo e datação arqueomagnética: princípios e métodos
}

\author{
Gelvam A. Hartmann* \\ Marisa C. Afonso ** \\ Ricardo I. F. Trindade*
}

\begin{abstract}
HARTMANN, G.A.; AFONSO, M.C.; TRINDADE, R.I.F. Arqueomagnetismo e datação arqueomagnética: princípios e métodos. Revista do Museu de Arqueologia e Etnologia, São Paulo, 17: 445-459, 2007.
\end{abstract}

Resumo: O campo magnético da Terra oscila em várias escalas de tempo tanto em direção quanto em intensidade. As oscilações na escala de tempo arqueológico $\left(1,7 \mathrm{X} 10^{3}\right.$ a $1 \mathrm{X} 10^{4}$ anos) são de origem interna (no núcleo externo, líquido e condutor), têm abrangência regional $\left(\sim 200.000 \mathrm{~km}^{2}\right)$ e correspondem à parte das variações seculares do campo geomagnético. $\mathrm{O}$ estudo dessas variações em diferentes partes do globo permite construir curvas de referência. Quando bem conhecidas, essas curvas podem ser utilizadas na datação arqueomagnética, que consiste na comparação de um dado arqueomagnético obtido em um artefato ou estrutura arqueológica com a curva de referência para a região em estudo. Neste trabalho são apresentados os princípios do arqueomagnetismo, as formas como o campo é registrado em material arqueológico, as técnicas para obtenção de dados arqueomagnéticos e os métodos utilizados na datação arqueomagnética. Além disso, são apresentadas as perspectivas para o arqueomagnetismo no Brasil e a aplicação desta técnica de datação na porção meridional da América do Sul.

Palavras-chave: Artefatos cerâmicos - Estruturas de combustão - Pinturas rupestres - Arqueomagnetismo - Datação arqueomagnética.

\section{Introdução}

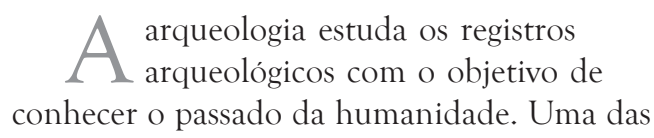

$\left({ }^{*}\right)$ Departamento de Geofísica, Instituto de Astronomia, Geofísica e Ciências Atmosféricas, Universidade de São Paulo. G.A.H. (gelvam@iag.usp.br); R.I.F.T. (rtrindad@iag.usp.br) $\left.{ }^{* *}\right)$ Museu de Arqueologia e Etnologia, Universidade de São Paulo. (marisa@br2001.com.br) informações mais importantes para este tipo de estudo é a idade em que determinados eventos ocorreram. Para isso, existem dois tipos de datação: a datação relativa e a datação absoluta. $\mathrm{Na}$ datação relativa, os artefatos, fósseis e sítios são relacionados a uma escala temporal que é baseada nas correlações estratigráficas, nos processos estilísticos ou na seqüência evolucionária dos indivíduos em estudo. Além disso, a cronologia pré-histórica humana pode ser balizada pelo registro de eventos geológicos recentes (Período Quaternário), como varia- 
ções climáticas, que por sua vez podem ser inferidas e servir de marcadores temporais. A datação absoluta é o processo que determina uma idade específica para um sítio ou artefato arqueológico. A datação absoluta utiliza-se de propriedades físicas ou químicas dos materiais em estudo para definir um ponto ou intervalo no tempo. A determinação do intervalo de tempo em que os processos físicos ocorreram indica quando os artefatos ou fósseis de interesse foram produzidos ou abandonados. Em geral, as técnicas de datação absoluta se utilizam de curvas de calibração, que dependem da região do globo em que os vestígios foram submetidos aos processos físicos, como, por exemplo, a datação por $\mathrm{C}^{14}$, por termoluminescência e por dendrocronologia. Outra técnica de datação que utiliza curvas de calibração é o arqueomagnetismo, no qual as variações de direção (declinação e inclinação) e intensidade do campo magnético da Terra no passado arqueológico e geológico servem como marcos temporais para a datação de artefatos e estruturas arqueológicas.

O campo magnético da Terra é definido, em cada ponto, a partir de sua declinação $(D$, ângulo do vetor campo magnético com relação ao norte), inclinação ( $I$, ângulo do vetor campo magnético com relação ao plano horizontal) e intensidade $(H$, módulo do vetor campo magnético). O campo magnético da Terra é bastante complexo e apresenta uma grande variabilidade, em escalas de tempo que vão de milisegundos a milhões, ou mesmo bilhões de anos (e.g., Merrill et al. 1998). As variações de curto período decorrem de flutuações no fluxo de correntes na ionosfera e magnetosfera, da variação diurna e de tempestades magnéticas. Estas variações são detectadas a partir de dados de observatórios magnéticos, ativos desde meados do século XIX e, mais recentemente, por dados de satélites (e.g., MAGSAT, OERSTED e CHAMP). As variações mais lentas, por outro lado, são decorrentes essencialmente da atividade interna do planeta, pela movimentação do fluido condutor no núcleo externo da Terra. Estas variações são chamadas de "variação secular" do campo magnético da Terra, que atuam na escala de anos a milhares de anos. A Figura 1 exemplifica como a declinação do campo magnético da Terra varia no tempo e no espaço. As variações de longo período compreendem as excursões e também as reversões do campo. Durante as reversões, ocorre uma inversão entre o norte e o sul magnético, isto é, a declinação do campo varia de $180^{\circ}$. As excursões correspondem a mudanças importantes na declinação, mas que não resultam em uma inversão completa. Esses fenômenos têm duração de aproximadamente 5.000 anos e podem apresentar uma periodicidade de dezenas de milhares de anos até dezenas de milhões de anos. Nessas escalas de tempo, diversas bases de dados, advindas dos observatórios geomagnéticos e de dados obtidos em materiais arqueológicos e em rochas, têm fornecido informações importantes quanto às variações direcionais do campo e, em menor grau, sobre as variações na intensidade do campo que as acompanham. Neste contexto, a porção Sul do globo contribui com menos de $5 \%$ dos dados da base mundial, sendo que somente $1 \%$ dos dados provém da América do Sul (Perrin e Schnepp 2004).

O estudo das variações temporais do campo magnético da Terra depende da base de dados utilizada. As variações na escala temporal de $1 \times 10^{\circ}$ a $5 \times 10^{2}$ anos (no máximo) são conhecidas como 'variações do campo no período histórico', variações da ordem de $1,7 \times 10^{3}$ a $1 \times 10^{4}$ anos são conhecidas como 'variações arqueomagnéticas' e variações da ordem de $7,8 \times 10^{5}$ e $5 \times 10^{6}$ anos são conhecidas como 'variações paleomagnéticas' (Carlut et al. 1999). Atualmente, dados arqueomagnéticos preenchem uma lacuna importante que existe entre os campos histórico e paleomagnético.

$\mathrm{O}$ arqueomagnetismo é o estudo do campo magnético da Terra em escalas de tempo arqueológico. O conhecimento dessas variações em uma dada região pode ser utilizado como uma ferramenta de datação. A chamada "datação arqueomagnética" é uma técnica de datação baseada em dois fenômenos físicos: a variação secular do campo magnético da Terra para uma determinada região do globo e a capacidade de certos materiais arqueológicos registrarem uma magnetização remanente estável quando expostos ao campo magnético 


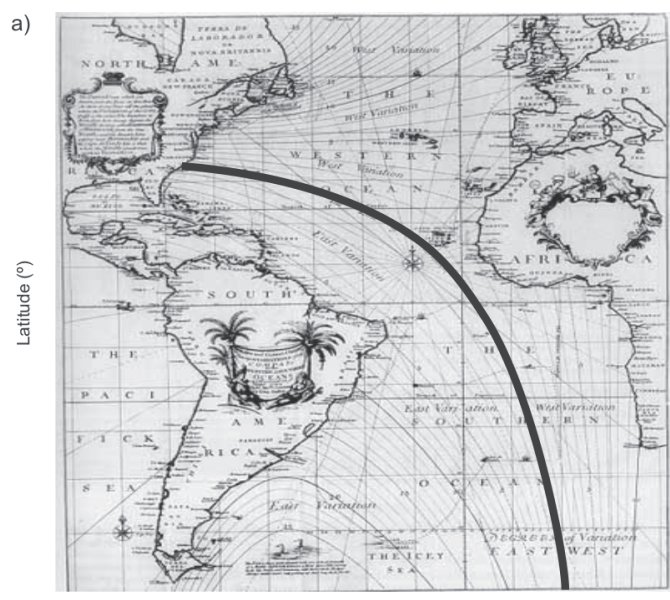

Longitude $\left({ }^{\circ}\right)$

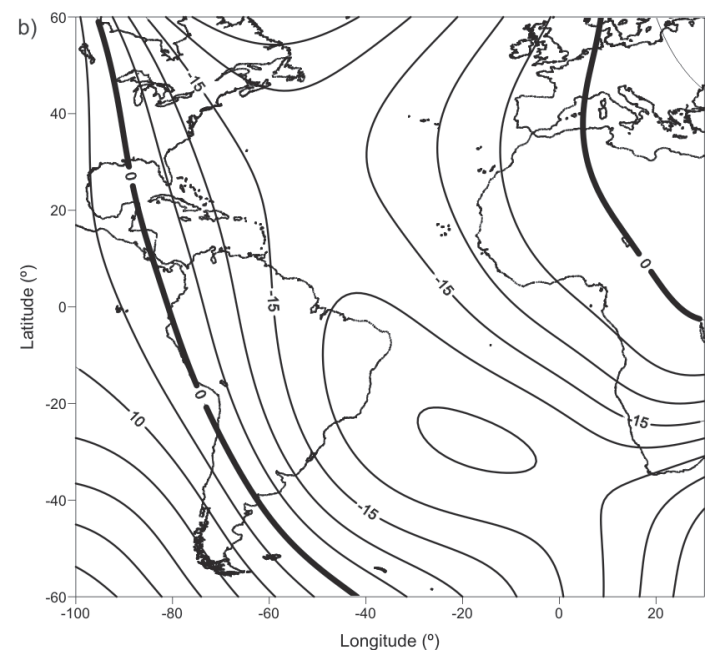

Fig. 1. Mapas de declinação magnética. Em (a) o mapa com linhas de mesma declinação construído para o ano 1700 AD por Halley (modificado de Courtillot e LeMöuel 2007). Em (b) o mapa de 2005 obtido a partir do modelo do International Geomagnetic Reference Field. Observe que em 305 anos a linha que indica declinação zero (linha grossa em ambos os mapas) migrou mais de $5.000 \mathrm{~km}$ para oeste.

da Terra no momento da produção ou descarte (Aitken 1999). O princípio desta datação reside na comparação de um dado de direção ou intensidade obtido em materiais arqueológicos de idade desconhecida, com o valor de direção e/ou intensidade da curva de variação do campo magnético da Terra para a região de estudo, conhecida também como "curva de referência” (e.g., Le Goff et al. 2002). Neste trabalho, serão apresentados as formas de registro do campo magnético terrestre em materiais arqueológicos e os métodos para aquisição de dados arqueomagnéticos. Por fim, será apresentada a técnica para determinação de idades arqueomagnéticas, aplicada atualmente com sucesso na Europa, e as perspectivas do arqueomagnetismo no Brasil.

\section{Aquisição de magnetização em materiais arqueológicos e geológicos}

Os artefatos e estruturas arqueológicas, as rochas e os sedimentos, contêm pequenas frações de minerais magnéticos (e.g., magnetita, hematita) que têm a capacidade de reter uma magnetização remanente. Essa magnetização pode ser registrada de duas formas (Figura 2): (i) durante o aquecimento e resfriamento de um material cerâmico ou estrutura de combustão, ou (ii) durante a deposição de partículas magnéticas em pinturas, cimentos ou sedimentos. A remanência magnética associada com o evento arqueológico estudado é conhecida como magnetização remanente primária. A magnetização remanente natural (MRN) do material consiste de uma magnetização primária somada a uma ou mais magnetizações secundárias. As magnetizações secundárias indicam eventos que muitas vezes não têm relação com o evento original de formação ou produção do material estudado (e.g., Butler 1998).

Determinados tipos de material arqueológico podem preservar tanto o registro de direção quanto a intensidade do campo. Eles correspondem aos materiais que sofreram aquecimento, como por exemplo, cerâmicas, tijolos, telhas, utensílios cerâmicos, argila cozida e estruturas de combustão (fornos para tijolos, fornalhas, fornos domésticos, pisos de lareiras e pisos cerâmicos). Após o aquecimento, os materiais resfriam abaixo de uma temperatura crítica (temperatura de bloqueio) e então os minerais magnéticos registram uma magnetização que é paralela e proporcional ao 

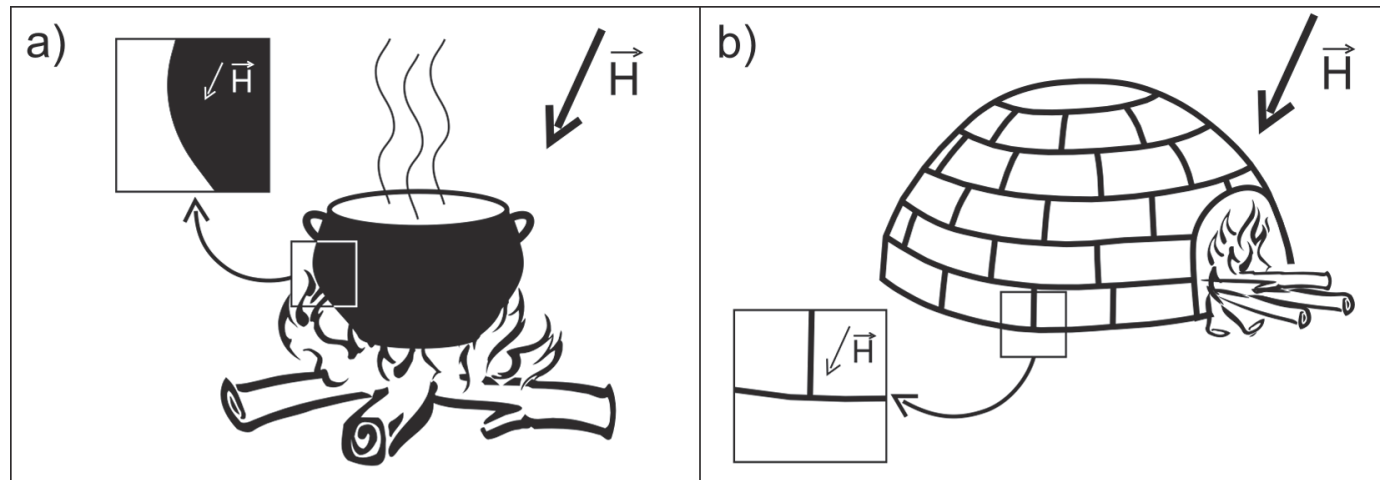

c)

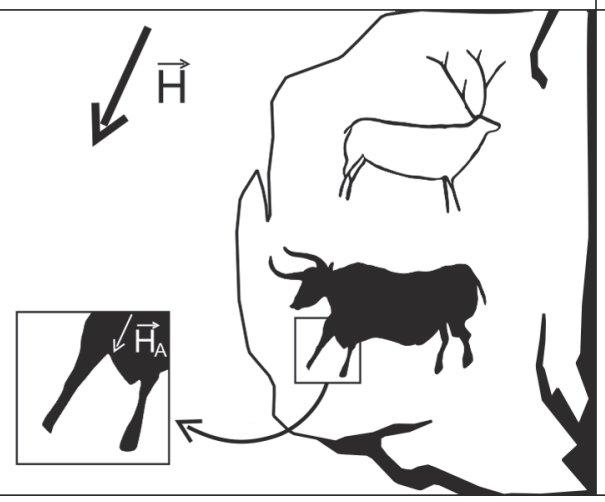

d)

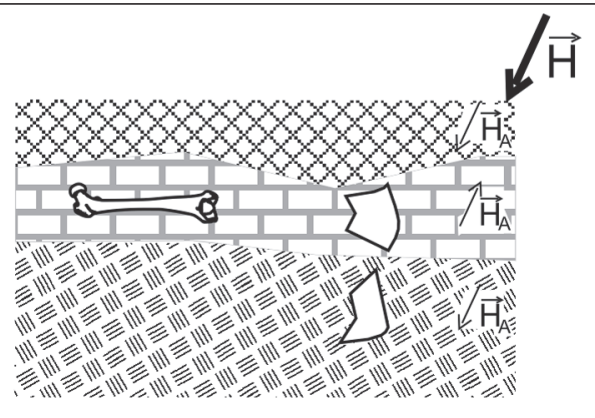

Fig. 2. Processos de aquisição de magnetização remanente em contextos arqueológicos. Em (a) a magnetização é adquirida pelo aquecimento de um utensílio doméstico. Em (b) a magnetização é adquirida pelo aquecimento em um forno cerâmico. Nesses dois exemplos os materiais adquirem uma magnetização térmica. No caso do forno (estrutura arqueológica), além da intensidade do campo $(H)$, o material pode ser utilizado para determinar a orientação do campo magnético (D e I). Em (c) a magnetização é registrada durante a secagem da tinta em uma pintura rupestre. A orientação do campo magnético no momento em que a pintura foi efetuada fica registrada pelas partículas magnéticas contidas no pigmento. Em (d) observa-se um sítio arqueológico com diferentes níveis estratigráficos. Nota-se que as direções do campo antigo $\left(H_{A}\right)$ são diferentes para os diferentes níveis estratigráficos. Pode-se então correlacionar estas variações com o material arqueológico encontrado nos estratos. Tanto em (c) quanto em (d) apenas a informação da direção do campo antigo pode ser recuperada.

campo magnético da Terra da época. Essa magnetização é chamada de magnetização remanente térmica (e.g., Dunlop e Özdemir 1997). Cada vez que o material é reaquecido, ele adquire uma nova magnetização térmica, de forma que o evento registrado no material é aquele do último aquecimento/resfriamento. Assim, telhas, tijolos e cerâmicas que não sofreram reaquecimento preservam a magnetização térmica adquirida no momento da produção. Outros materiais, como as estruturas de combustão, registram a magnetização térmica do último aquecimento/resfriamento, que em geral está associada ao abandono. Do mesmo modo, as lavas de erupções vulcânicas históricas adquirem uma magnetização térmica após resfriarem na presença do campo magnético terrestre. Deste modo, uma seqüência de fluxos de lava pode também ser usada para a construção de curvas de referência arqueomagnéticas (Tanguy et al. 2003). Estes materiais fornecem a intensidade do campo magnético da Terra registrada durante o seu último aquecimento. Com as estruturas de combustão pode-se também determinar a declinação e inclinação do campo no momento da última queima se essas estruturas não foram movimentadas posteriormente. Tijolos e telhas podem 
fornecer dados de inclinação magnética caso tenham sido queimados no plano horizontal, o que nem sempre é possível de determinar. As Figuras 2a e 2b ilustram o registro do campo magnético da Terra através dos processos de aquecimento.

Materiais que não foram submetidos a temperaturas elevadas também têm a capacidade de registrar informações sobre o campo magnético da Terra. Pinturas rupestres e cimentos podem adquirir magnetização estável. Nesses materiais, as partículas magnéticas rotacionam e alinham-se com o campo magnético da Terra, fixando-se nesta posição quando a pintura ou o cimento secam. Esta magnetização é conhecida como magnetização remanente pictórica (Chiari e Lanza 1997, 1999; Zanella et al. 2000; Goguitchaichvili et al. 2004). Neste caso, o evento que corresponde ao registro magnético é a aplicação da pintura ou do cimento. Os sedimentos depositados em fundos de lagos e cavernas podem adquirir uma magnetização deposicional, em um mecanismo muito semelhante ao observado nas pinturas murais e nas pinturas rupestres. Esses materiais fornecem um registro das variações direcionais do campo magnético da Terra e podem, eventualmente, ser utilizados para construir curvas de variação relativa da intensidade do campo (e.g., Valet 2003). As Figuras $2 \mathrm{c}$ e $2 \mathrm{~d}$ mostram como esses processos ocorrem em um sítio arqueológico.

Uma parte da magnetização primária, adquirida durante o resfriamento de artefatos arqueológicos ou de rochas, decai com o tempo mesmo na presença de campos muito fracos como no caso do campo magnético da Terra. Ao decair, esta parte do registro magnético tende a se alinhar com o campo magnético atual gerando uma magnetização viscosa.

Outros processos que alteram a magnetização original envolvem a exposição dos materiais a fortes campos magnéticos ou fortes correntes elétricas, como na presença de raios. Nesse caso as amostras adquirem uma magnetização remanente isotérmica. Magnetizações secundárias podem também ser adquiridas por transformações químicas resultantes da alteração dos minerais magnéticos durante o soterramento.
Em todos esses casos, os procedimentos de desmagnetizações sucessivas permitem eliminar a contribuição secundária e isolar a magnetização primária em rochas e artefatos arqueológicos.

\section{Métodos para determinação da direção e intensidade do campo arqueomagnético}

As variações do campo magnético da Terra tanto em intensidade quanto em direção são obtidas a partir do registro magnético em rochas e artefatos arqueológicos. A magnetização remanente é paralela e proporcional ao campo magnético na época de produção ou abandono do objeto arqueológico em estudo. Embora se baseiem em princípios semelhantes, as técnicas para obtenção de dados de intensidade e dados direcionais são diferentes. Abaixo apresentamos os procedimentos para coleta, preparação, medida e análise das amostras.

\subsection{Coleta e preparação de amostras}

A amostragem depende do tipo de registro que se pretende obter, além do tipo e quantidade de material necessário para o tratamento de laboratório. Para a determinação somente da intensidade magnética as amostras não precisam ser orientadas, como é o caso de fragmentos cerâmicos, mas deve-se conhecer o seu local de origem. Por outro lado, para obtenção de dados direcionais, as amostras precisam ser orientadas na mesma posição na qual se deu a sua produção ou o abandono, no caso de estruturas arqueológicas. Para alguns materiais cuja posição de produção pode ser inferida, como telhas e tijolos, podese obter a inclinação do campo magnético terrestre mesmo que esses objetos tenham sido deslocados após a aquisição da magnetização. A orientação das amostras é feita com o auxílio de uma bússola magnética ou de uma bússola solar. Para uso da bússola magnética deve-se atentar para o fato da existência de anomalias magnéticas locais, que podem interferir nas medidas. A orientação deve ser feita em uma das faces da amostra da seguinte forma (Figura 3): inicialmente, utilizando um 
nível, identifica-se uma linha horizontal na face escolhida para orientação; essa linha deve ser marcada com uma seta na amostra; obtémse então, com a bússola, a orientação dessa linha com relação ao norte geográfico; em seguida obtém-se a inclinação da face da amostra na orientação normal à seta.

O vetor magnetização de uma estrutura ou artefato arqueológico é determinado a partir da média de várias medidas. Portanto, num caso ideal deve-se trabalhar com pelo menos dez amostras de um mesmo objeto para obter uma boa estimativa da orientação e intensidade do campo magnético antigo. $\mathrm{O}$ tamanho das amostras depende da homogeneidade do material a ser amostrado, de sua disponibilidade em função de restrições de preservação e de abundância. Nos casos em que não há restrições para a amostragem podem-se coletar blocos com cerca de $10 \times 10 \times 5 \mathrm{~cm}$, que são cortados no laboratório em amostras cúbicas de $1 \times 1 \times 1 \mathrm{~cm}$. Em situações nas quais há maior restrição para a retirada de amostras do sitio, podem-se coletar cilindros com
$2,5 \mathrm{~cm}$ de diâmetro ou cilindros com $1 \mathrm{~cm}$ de diâmetro, utilizando-se uma broca diamantada. Nos casos onde há muito pouco material disponível para amostragem, coletam-se fragmentos menores do que $1 \mathrm{~cm}$ de diâmetro que são "encapsulados" em alumina ou sal desidratado e moldados na forma de cubos ou cilindros. Amostras de pinturas rupestres e murais são orientadas na face da pintura (procedimento semelhante ao da Figura 3) e extraídas com o auxílio de fita dupla-face.

\section{$3.2 \mathrm{O}$ processo de desmagnetizaç̃es sucessivas}

Para determinação de um valor de intensidade ou direção de magnetização que represente a orientação do campo antigo, deve-se separar a magnetização primária das magnetizações secundárias. Para separação destas componentes utiliza-se a técnica desmagnetização por passos sucessivos de campos alternados ou temperatura (e.g., Butler 1998). Essas técnicas se baseiam no fato de que os grãos magnéticos a)

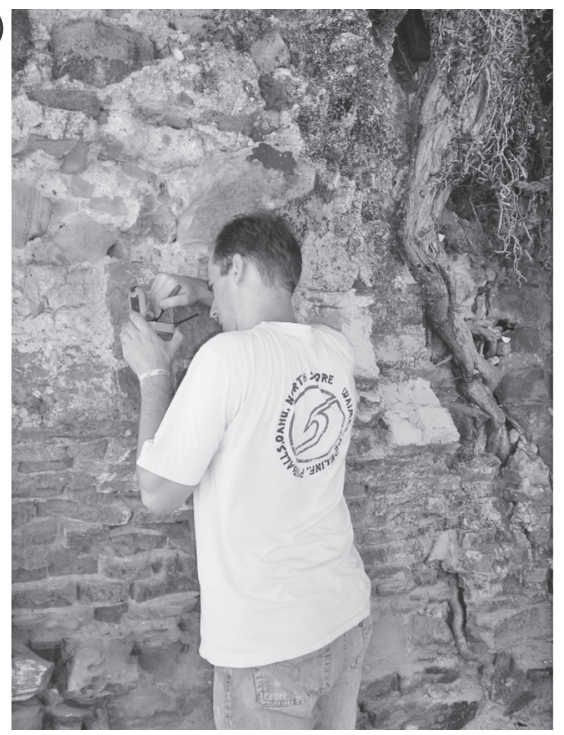

b)

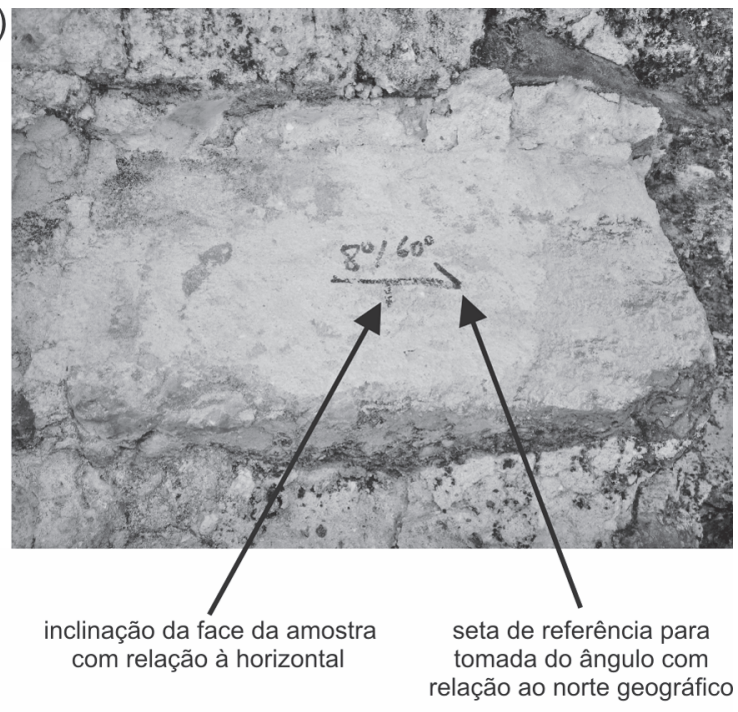

Fig. 3. Coleta e orientação de amostras arqueomagnéticas. Em (a), marcação da orientação de um tijolo de um forno arqueológico em Itaparica (BA) com auxílio de uma bússola magnética. Em (b) estão indicadas as marcações de orientação. A seta corresponde a uma linha horizontal e a linha menor, perpendicular à seta, indica a direção de máxima inclinação da face que foi orientada. Determina-se o ângulo entre a seta e o norte (azimute) e o ângulo entre a face amostrada e a horizontal (mergulho do plano). Ambos os ângulos são marcados na própria amostra. A orientação é feita antes da retirada do material do local. 
mais estáveis, que guardam a magnetização primária, apresentam valores de coercividade e temperaturas de bloqueio mais elevadas. Portanto, ao desmagnetizar as frações de baixa coercividade ou de baixa temperatura de bloqueio estamos "limpando" o sinal das componentes de magnetização secundárias. $\mathrm{Na}$ desmagnetização por campos alternados a amostra é submetida a campos magnéticos alternados cada vez mais elevados. Esses campos variam desde um valor de pico até zero. Assim, elimina-se a magnetização dos grãos com coercividade mais baixa do que o valor de pico do campo alternado. Repetindo-se esse procedimento até campos da ordem de $100 \mathrm{mT}$ (militesla) pode-se isolar o sinal dos grãos com alta coercividade. $\mathrm{Na}$ desmagnetização térmica, as amostras são submetidas ao aquecimento até uma determinada temperatura e resfriadas em campo magnético nulo. Durante esse processo os grãos com temperatura de bloqueio inferior à temperatura do passo de aquecimento perdem sua magnetização original. Este processo é repetido até temperaturas da ordem de $700^{\circ} \mathrm{C}$. Em geral, as componentes secundárias são removidas em torno de 5 a $20 \mathrm{mT}$ para a técnica utilizando campos alternados ou $200^{\circ} \mathrm{C}$ a $450^{\circ} \mathrm{C}$ para a técnica de aquecimentos sucessivos.

\subsection{Determinação da direção do campo magnético}

A direção da magnetização primária em cada amostra é obtida por meio de gráficos vetoriais ortogonais, conforme ilustra a Figura 4a. Neste exemplo a magnetização secundária, indicada pela mudança de orientação nos diagramas, é separada logo nos primeiros passos de desmagnetização. A análise por componentes principais é usada para ajustar o segmento linear da curva que corresponde à magnetização primária (Kirschvink 1980). Assim, a direção de melhor ajuste e o erro correspondente são então calculados para esta componente. Outra forma de visualizar os mesmos dados da Figura 4a é através da projeção estereográfica, como mostra a Figura 4b. Após as direções serem individualmente determinadas em todas as amostras de um determinado sítio, a direção média pode ser calculada pela soma vetorial das direções individuais. Inicialmente são calculadas as médias das amostras em estruturas ou fragmentos. Em seguida, são calculadas as médias das estruturas ou fragmentos do mesmo sítio. Utiliza-se no cálculo da direção média a estatística desenvolvida por Fischer (1953) que permite também estimar a precisão e confiabilidade da estimativa a partir dos parâmetros á (cone de erro) e o $k$ (parâmetro de concentração). Valores altos de $k$ e baixos de á ${ }_{95}$ indicam dados direcionais confiáveis.

\subsection{Determinação da intensidade do campo magnético}

A paleointensidade do campo magnético da Terra é obtida pelo método desenvolvido por Thellier e Thellier (1959) para estudos de cerâmicas arqueológicas, podendo ser aplicado também a qualquer material cuja magnetização tenha origem em um aquecimento. Atualmente, os experimentos de paleointensidade usam versões modificadas do método ThellierThellier original. Entre as mais conhecidas e usadas estão o método Coe (1967) e o método Aitken et al. (1988). Experimentalmente, a determinação da intensidade é muito mais complicada do que a determinação da orientação do campo magnético.

A intensidade da magnetização térmica adquirida por uma rocha ou artefato cerâmico durante o resfriamento é proporcional à intensidade do campo indutor antigo desde que este seja suficientemente baixo (da ordem de algumas dezenas de microteslas). $\mathrm{O}$ método se baseia na comparação da magnetização natural $M_{T}$ com uma magnetização $M_{L}$ de laboratório produzida em um campo conhecido $H_{L}$ (campo de laboratório). O campo antigo $H_{A}$ pode então ser determinado segundo,

$$
H_{A}=H_{L} \frac{M_{T}}{M_{L}}
$$


a)

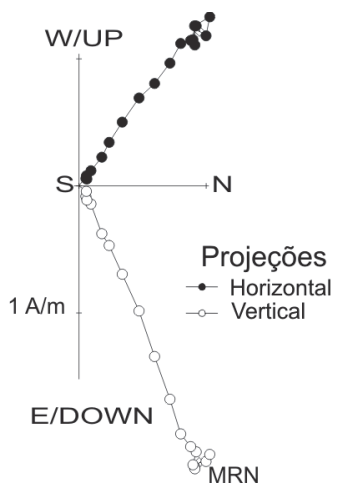

c)

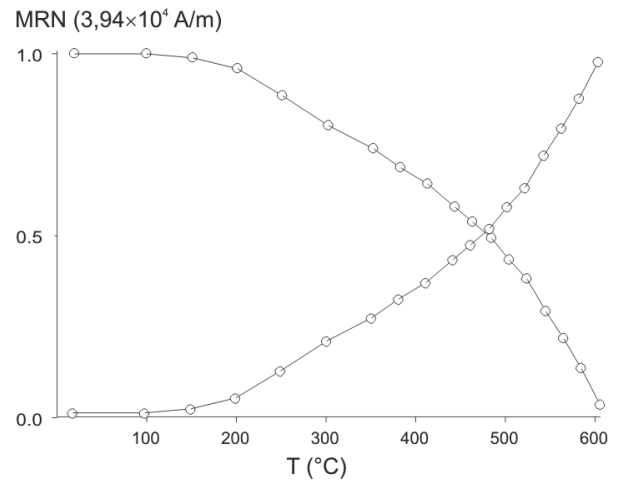

b)

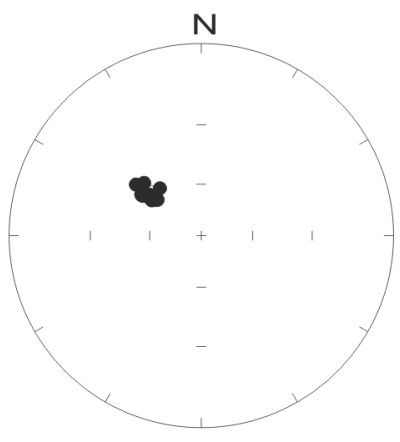

d)

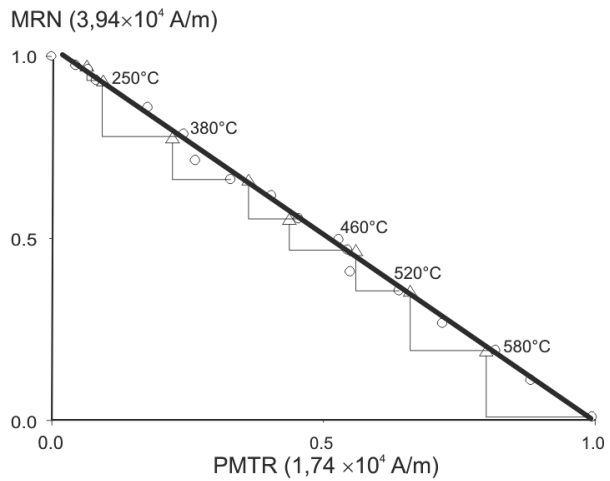

Fig. 4. Representação dos dados de direção e intensidade do campo magnético terrestre. A Figura (a) é um exemplo que mostra as projeções ortogonais obtidas para uma amostra. As duas curvas correspondem à projeção do vetor magnetização nos planos horizontal (símbolos cheios) e vertical (símbolos vazados) após cada etapa de desmagnetização. A Figura (b) mostra os mesmos dados apresentados em (a), mas em projeção estereográfica. Nesse diagrama a orientação do vetor após cada etapa de desmagnetização é representada como um ponto na rede estereográfica equi-área. A partir desses diagramas são obtidos os dados direcionais ( $D$ e $I$ ) de uma amostra. A Figura (c) representa um experimento do tipo Thellier-Thellier. Neste experimento, ao mesmo tempo em que a magnetização natural do objeto é removida, uma magnetização térmica de laboratório é implantada com um campo magnético de intensidade conhecida. O diagrama mostra a evolução da magnetização removida e da magnetização artificial adquirida. A Figura (d) corresponde a um diagrama de Arai para os mesmos dados de (c). Nesse diagrama representa-se no eixo vertical a magnetização removida e no eixo horizontal a magnetização adquirida em laboratório. $\mathrm{O}$ valor da intensidade do campo registrado na amostra é dado pelo ajuste da reta por mínimos quadrados.

De acordo com a equação (01), a razão entre a magnetização natural e a magnetização de laboratório deve corresponder a uma reta, cuja inclinação é utilizada para determinação de $H_{A}$.

Há várias formas possíveis de substituir progressivamente a magnetização natural por uma magnetização de laboratório. A mais utilizada atualmente é aquela desenvolvida por Coe (1967) e Aitken et al. (1988). Nesses proto- colos de medida a magnetização natural é progressivamente desmagnetizada num processo semelhante à desmagnetização térmica descrita anteriormente. Mas logo após cada passo de desmagnetização a amostra adquire uma magnetização de laboratório na mesma temperatura e com campo conhecido. Os duplos aquecimentos são repetidos em temperaturas cada vez mais elevadas até a 
completa substituição da magnetização natural pela magnetização de laboratório. Os resultados são apresentados na forma de gráficos da magnetização remanente natural (MRN) restante e da magnetização de laboratório (MTR) adquirida contra a temperatura (Figura 4c). Outra forma de apresentação dos dados (e a mais utilizada) é através do gráfico de Arai (Nagata et al. 1963), onde a MRN é representada no eixo vertical e a MTR é representada no eixo horizontal. A inclinação da reta neste diagrama corresponde à razão $-H_{A} / H_{L}$ e permite determinar a paleointensidade (Figura 4d).

Durante as medidas, alguns passos de temperatura podem ser repetidos para determinar se há variação na capacidade de registrar remanência das amostras. Estes passos são chamados de "checagem das magnetizações térmicas parciais" ( $p$ MTR checks). A diferença entre as duas magnetizações, em uma dada temperatura, indica uma mudança nas condições de aquisição de remanência térmica e, portanto, uma estimativa falsa do paleocampo. Além dos pMTR checks, são realizados também testes para determinação da anisotropia magnética e da taxa de resfriamento das amostras. Estes testes são muito importantes para determinação da intensidade em cerâmicas, como já pôde ser observado experimentalmente (e.g., Chauvin et al. 2000; Genevey e Gallet 2002).

\section{Datação arqueomagnética}

A datação arqueomagnética é realizada através da comparação de uma curva de referência para a variação secular com os dados arqueomagnéticos obtidos em artefatos de idade desconhecida (e.g., Aitken 1999). As curvas de referência revelam as variações em direção e intensidade do campo magnético da Terra. Os dados compilados para uma dada região são arbitrariamente corrigidos para um local de referência através da correção para o pólo geomagnético virtual (Daly e LeGoff 1996). Os dados de declinação e inclinação magnéticas para um determinado local são usados no cálculo da posição do pólo geomagnético virtual, que é então utilizado para calcular as declinações e inclinações esperadas para o local de referência. Este tipo de abordagem é válido apenas para uma determinada região do planeta. Atualmente, as curvas de referência estão disponíveis para os últimos 2.000 a 3.000 anos em várias regiões do planeta, incluindo a América do Norte (Stenberg 1989; Labelle e Eighmy 1997; Yu et al. 2000), América Central (Morales et al. 2001; Bowles et al. 2002; Soler-Arechalde et al. 2006), Leste da Ásia (Cong e Wei 1989), Leste e Oeste da Europa (Bucur 1994; Kovacheva 1997; Kovacheva et al. 1998; Chauvin et al. 2000; Genevey e Gallet 2002; Genevey et al. 2003; Kovacheva et al. 2004; Schnepp e Lanos 2005; Gómez-Paccard et al. 2006). Existem também compilações globais, como aquelas de Valet (2003); Perrin e Schnepp (2004) e Korte et al. (2005). No entanto, a área em que uma dada curva de referência pode ser aplicada à datação arqueomagnética é da ordem de $200.000 \mathrm{~km}^{2}$, visto que há sutis diferenças de variação do campo magnético da Terra para locais que são um pouco distantes entre si. Um exemplo deste tipo de variação pode ser observado nas Figuras $5 \mathrm{a}$ e 5b, que mostram uma comparação das curvas arqueomagnéticas para os últimos 3.000 anos, obtidas para a Ibéria, França e Alemanha (Gómez-Paccard et al. 2006). Neste exemplo observam-se as pequenas diferenças de declinação e inclinação quando se comparam locais diferentes, mesmo quando corrigidos para um local de referência. A Figura 5c mostra uma compilação dos dados de intensidade magnética obtidos para os últimos 3.000 anos em artefatos arqueológicos da França. Note que os dados de intensidade variam mais rapidamente do que os dados direcionais. Além de dados arqueomagnéticos, as curvas de referência podem ser construídas também com dados de observatórios, dados históricos (obtidos a partir de cartas de navegação) ou ainda dados paleomagnéticos. No entanto, a datação em amostras paleomagnéticas é feita por métodos radiométricos e, portanto, a precisão é diferente dos métodos utilizados para datação de materiais arqueológicos (Tanguy et al. 2003; McIntosh e Catanzariti 
2006). Além da diferença da precisão entre diferentes métodos de datação, um outro problema associado à construção das curvas de referência é a distribuição desigual dos dados tanto no tempo quanto no espaço. Alguns métodos estatísticos são utilizados para minimizar estes problemas, incluindo estatística bivariada com janelas temporais móveis (LeGoff et al. 2002) ou estatística Bayesiana, na qual o empilhamento estratigráfico local pode ser considerado (Lanos et al. 2005).

A determinação de uma idade arqueomagnética pressupõe que os dados magnéticos obtidos em um artefato ou estrutura arqueológica apresentem características semelhantes aos dados utilizados na curva de referência. Considerando-se os erros da curva de referência e os erros do dado arqueomagnético, estima-se a probabilidade de que ambos compartilhem o mesmo valor. A idade corresponde ao ponto no qual há maior probabilidade de concordância entre ambos. A Figura 6 mostra uma datação arqueomagnética realizada em um forno cerâmico localizado em uma vila próxima a Paris. Esta datação foi realizada utilizando a curva de referência da França, conforme ilustrado na Figura 5a. A curva de probabilidade, determinada pelo teste de Le Goff et al. (2002), indicou que a idade mais provável para a estrutura é em torno de 1010 \pm 35 AD. Este resultado está em concordância com outros vínculos temporais arqueológicos do sítio estudado. A qualidade da datação arqueomagnética depende: da qualidade da curva de referência (densidade e homogeneidade de dados), das taxas de variação do campo no período de interesse e da qualidade da determinação arqueomagnética nos artefatos ou estruturas que se deseja datar. Em geral as incertezas podem variar de \pm 25 a \pm 200 anos (McIntosh e Catanzariti 2006).

\section{Considerações finais}

As principais características do registro do campo magnético da Terra em materiais arqueológicos, bem como os métodos para aquisição de dados arqueomagnéticos foram descritos, utilizando exemplos para os últimos milhares de anos. Além disso, foram introduzidas as técnicas de análise estatística dos dados usados na datação arqueomagnética, que foi ilustrada com um exemplo de datação na França.

São importantes as formas como o campo magnético da Terra é registrado em materiais arqueológicos e como estas informações são recuperadas, pois indicam o tipo de dado a ser obtido (declinação e/ou inclinação e/ou intensidade). A magnetização pode ser obtida em materiais que sofreram aquecimento e que não foram aquecidos. Em geral, materiais que foram aquecidos em altas temperaturas e que não foram reaquecidos, além de poderem ser amostrados in situ, são os mais adequados para a determinação de dados direcionais e de intensidade. Materiais aquecidos e que não possuem orientação permitem apenas recuperar a intensidade magnética antiga. Materiais que não sofreram aquecimento fornecem apenas dados direcionais.

A datação arqueomagnética é obtida através da comparação de um dado arqueomagnético de idade desconhecida com a curva de referência para uma determinada região do globo. A datação arqueomagnética é, portanto, uma datação relativa, porque há a necessidade de um vínculo a priori (que neste caso é a curva de referência) para posterior comparação de um dado obtido em um artefato arqueológico. A datação arqueomagnética pode ser aplicada para regiões com curvas de referência bem definidas, que por sua vez podem ser construídas com outros tipos de dados, como dados de observatórios, históricos, arqueomagnéticos e paleomagnéticos. As curvas de referência utilizadas para datação arqueomagnética devem apresentar uma boa cobertura temporal e espacial dos dados. A precisão e confiabilidade da datação dependem de vários fatores, entre eles a qualidade da curva de referência e dos dados arqueomagnéticos usados para datação. As incertezas podem variar de \pm 25 a \pm 200 anos.

Os estudos arqueomagnéticos realizados até o momento indicam uma pobre cobertura espacial, principalmente para o hemisfério Sul do planeta. Neste sentido, o Brasil surge 


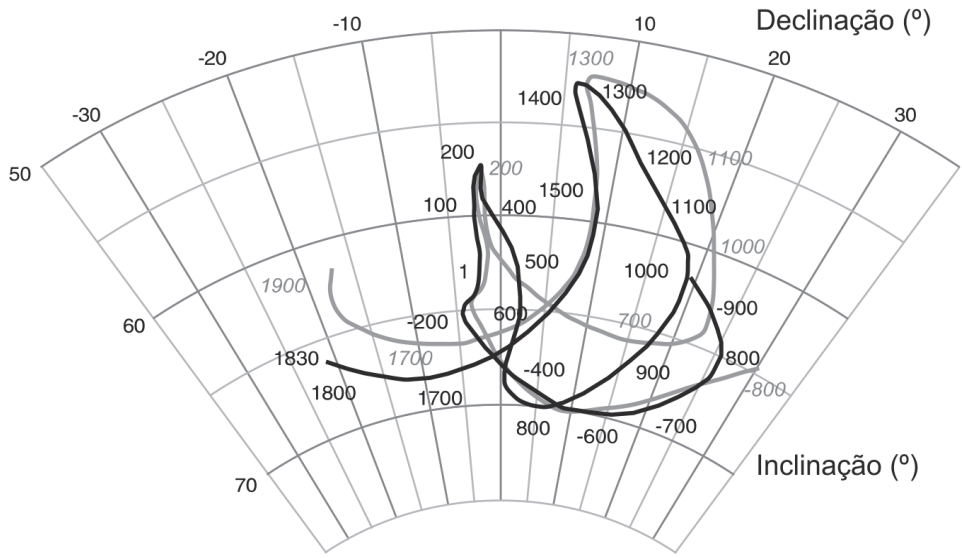

b) Curvas de variação secular para a Ibéria

) e Alemanha

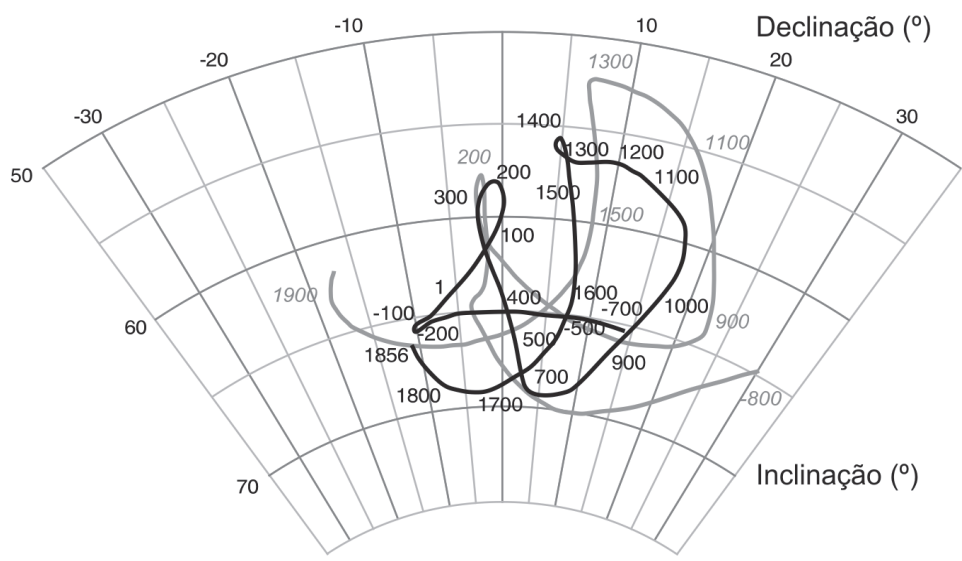

c) Curva de variação de intensidade para a França

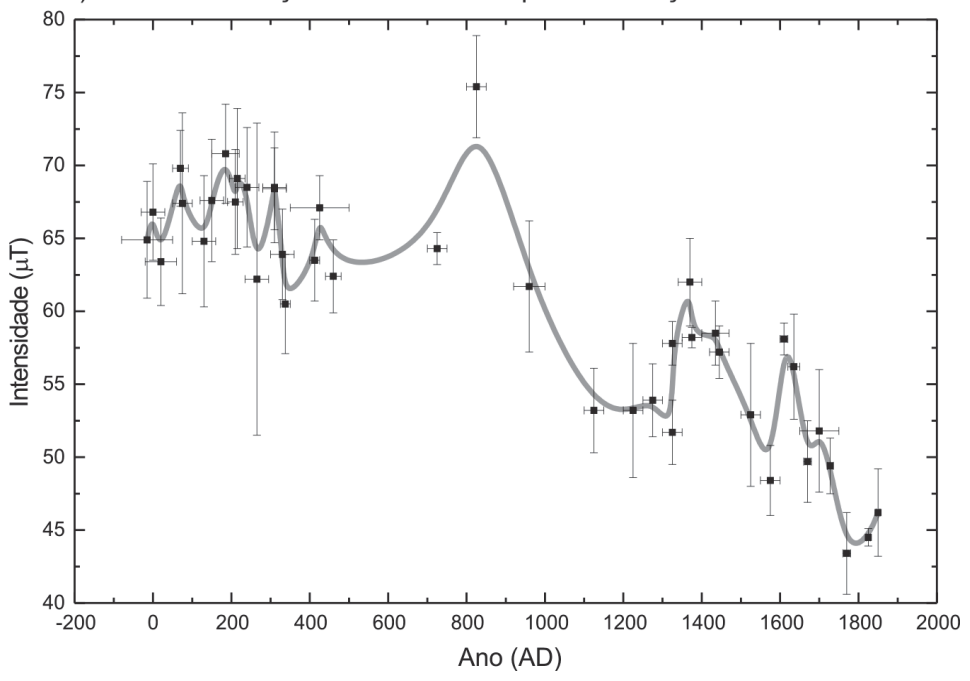

Fig. 5. As Figuras (a) e (b) mostram curvas de variação direcional ( $D$ e $I$ ) entre regiões da Europa. Em (a) as curvas da Ibéria e da França e em (b) as curvas da Ibéria e da Alemanha. Note que as diferenças entre as curvas são mais significativas entre a Ibéria e a Alemanha. Para fins de comparação todas as curvas foram reduzidas para a cidade de Madri. A Figura (c) mostra a variação da intensidade do campo magnético na França nos últimos 3.000 anos obtida a partir de estudos em cerâmicas. (curvas extraídas de GómezPaccard et al. 2006; Chauvin et al. 2000; Genevey e Gallet 2002; Gallet et al. 2005). 


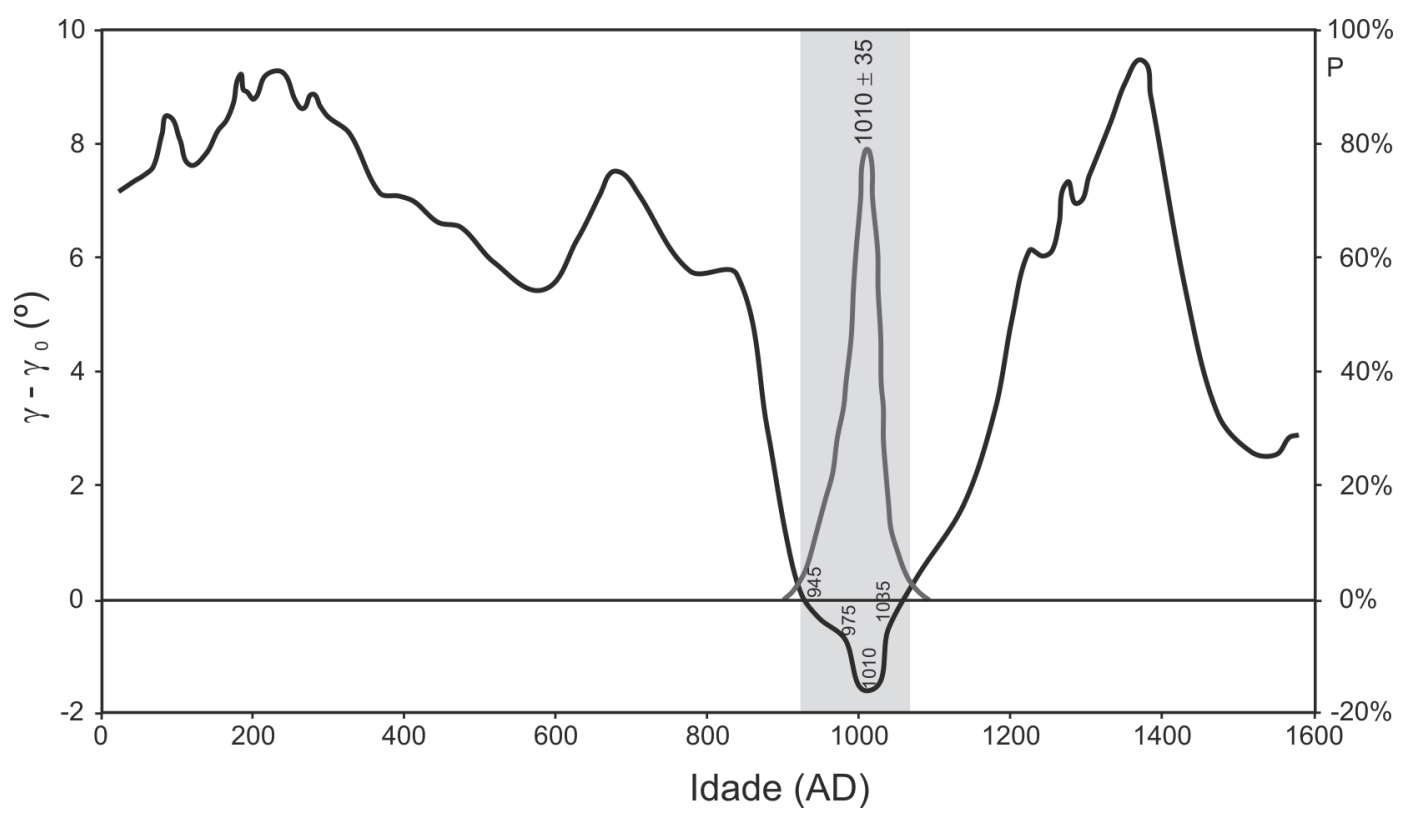

Fig. 6. Exemplo de uma datação arqueomagnética realizada em um forno da França. A datação utilizou a estatística bivariada e indicou um valor de 1010 \$35 AD, que está em concordância com o contexto arqueológico. A curva de referência utilizada para esta datação foi a curva de variação direcional para a França, mostrada na Figura 4a (figura modificada de Le Goff et al. 2002).

como um local importante para estudos de arqueomagnetismo. O projeto de arqueomagnetismo no Brasil vem sendo desenvolvido através de parceria firmada entre os grupos de paleomagnetismo do Instituto de Astronomia, Geofísica e Ciências Atmosféricas (Universidade de São Paulo) e do Institut de Physique du Globe de Paris e com os arqueólogos dos Museus de Arqueologia e Etnologia da Universidade de São Paulo e da Universidade Federal da Bahia. Os primeiros resultados de intensidade magnética obtidos em fragmentos cerâmicos coloniais e pré-coloniais do estado de São Paulo foram apresentados recentemente (Hartmann et al. 2007a, 2007b). Os dados obtidos apontam para a construção de uma curva de referência de boa qualidade para o Sudeste do Brasil. Esse trabalho resultará em breve na construção de uma curva de referência para a porção sul da América do Sul, abrindo a possibilidade de utilização do arqueomagnetismo como uma ferramenta alternativa para datação de artefatos arqueológicos dessa região.

\section{Agradecimentos}

Os autores agradecem ao MAE/USP e ao IAG/USP, pelo apoio institucional, à FAPESP (bolsa de doutorado 05/57782-4) e ao CNPq (bolsas de produtividade e pesquisa), pelo apoio financeiro, e aos Professores José Luiz de Morais (MAE/USP) e Carlos Etchevarne (MAE/UFBA) pelas amostras arqueológicas de projetos por eles coordenados. Lucimara Vianna e Lelis Melo do setor gráfico do IAG/USP foram os responsáveis pela elaboração da Figura 2. 
HARTMANN, G.A.; AFONSO, M.C.; TRINDADE, R.I.F. Archaeomagnetism and archaeomagnetic dating: principles and methods. Revista do Museu de Arqueologia e Etnologia, São Paulo, 17: 445-459, 2007.

\begin{abstract}
The direction and intensity of the Earth's magnetic field oscillates in different time-scales. Variations within $1,7 \mathrm{X} 10^{3}$ to $1 \times 10^{4}$ years are referred to as archaeomagnetic variations. They correspond to a part of the geomagnetic secular variation, have an internal origin (in the conductive fluid outer core), and are coherent over regions of $\sim 200.000 \mathrm{~km}^{2}$. The study of the archaeomagnetic variations in different parts of the globe enables the construction of regional master curves. Well-constrained master curves allow the use of archaeomagnetic variations as a dating tool. The archaeomagnetic dating corresponds to the comparison of an archaeomagnetic data obtained from an archaeological artifact or structure to the master curve for the region of interest. In this paper, we present the basis of archaeomagnetism, the mechanisms by which archaeological artifacts, structures and geological material record the ancient magnetic field, and how the magnetic signal is measured and interpreted, as well as the principles of archaeomagnetic dating. Also discussed are the perspectives for archaeomagnetic studies in Brazil and its application as a dating technique in southern South America.
\end{abstract}

Keywords: Ceramic artifacts - Burnt structures - Mural paintings Archaeomagnetism - Archaeomagnetic dating.

\title{
Referências bibliográficas
}

AITKEN, M.J.

1999 Archaelogical dating using physical phenomena. Rep. Prog. Phys., 62: 1333-1376.

AITKEN, M.J.; ALLSOP, A.L.; BUSSERLL, G.D.; WINTER, M.B.

1988 Determination of the intensity of the Earth's magnetic field during archaeological times: Reability of the Thellier technique. Rev. Geophys., 26: 3-12.

BOWLES J.; GEE J.; HILDEBRAND J.; TAUXE L.

2002 Archaeomagnetic intensity results from California and Ecuador: evaluation of regional data. Phys. Earth Planet. Inter., 203 (3-4): 967-981.

BUCUR, I.

1994 The direction of the terrestrial magnetic field in France, during the last 21 centuries. Recent progress. Phys. Earth Planet. Int., 87: 95-109.

BUTLER, R.F.

1998 Paleomagnetism: magnetic domains to geologic terranes. Tucson: Department of Geosciences, University of Arizona.

CARluT, J.; COURTILlOT, V.; HUlOT, G.

1999 Over how much time should the geomagnetic field be averaged to obtain the mean-paleomagnetic field? Terra Nova, 11: 239-243.

CHAUVIN, A.; GARCIA, Y.; LANOS, PH.; LAUBENHEIMER, F.

2000 Paleointensity geomagnetic field recovered on archaeomagnetic sites from France. Phys. Earth Planet. Int., 120: 111-136.

CHIARI, G.; LANZA, R.

1997 Pictorial remanent magnetization as an indicator of secular variation of the Earth's magnetic field. Phys. Earth Planet. Int., 101: 79-83.

1999 Remanent magnetization of mural paintings from the Bibliotheca Apostolica (Vatican, Rome). J. App. Geophys., 41: 137-143. 
COE, R.S.

1967 The determination of paleo-intensities of the Earth's magnetic field with emphasis on mechanisms which could cause nonideal behavior in Thelier's method. J. Geomag. Geoelectric., 19: 157-179.

CONG, Y.Z; WEI, Q.Y.

1989 Study of secular variation (2000 BC $1900 \mathrm{AD}$ ) based on comparison of contemporaneous records in marine sediments and baked clays. Phys. Earth Planet. Int., 56: 79-85.

COURTILLOT, V.; LEMÖUEL, J-L.

2007 The study of Earth's magnetism (1269 1950): a foundation by Peregrinus and subsequent development of Geomagnetism and Paleomagnetism. Rev. Geophys., 45 (RG3008): 1-31.

DALY, L.; LE GOFF, M.

1996 An updated and homogeneous world secular variation data base.1. Smoothing of the archaeomagnetic results. Phys. Earth Planet. Int., 93: 159-190.

DUNLOP, D.; ÖZDEMIR, Ö.

1997 Rock Magnetism - Fundamentals and Frontiers, New York: Cambridge University Press.

FISCHER, R.A.

1953 Dispersion on a sphere. Proc. Roy. Soc. London, A217: 295-305.

GALLET, Y.; GENEVEY, A.; FLUTEAU, F.

2005 Does Earth's magnetic field secular variation control centennial climate change? Earth Planet. Sci. Let., 236: 339-347.

GENEVEY, A.; GALLET, Y.

2002 Intensity of the geomagnetic field in western Europe over the past 2000 years: New data from ancient French pottery. J. Geophys. Res., 107(B11) article number: 2285.

GENEVEY, A.; GALLET, Y.; MARGUERON, J.C.

2003 Eight thousand years of geomagnetic field intensity variations in the eastern Mediterranean. J. Geophys. Res., 108 (B5) article number: 2228.

GOGUITCHAICHVILI, A.; SOLER, A.M.;

ZANELLA, E.; CHIARI, G.; LANZA, R.; URRUTIA-FUCUGAUCHI, J; GONZALEZ, T.

2004 Pre-columbian mural paintings from Mesoamerica as geomagnetic field recorders. Geophys. Res. Lett., 31: L12607.

GÓMEZ-PACCARD, M.; CHAUVIN, A.; LANOS,

PH.; THIRIOT, J.; JIMÉNEZ-CASTILLO, P.

2006 Archaeomagnetic study of seven contemporaneous kilns from Murcia (Spain). Phys. Earth Planet. Int., 157: 16-32.
HARTMANN, G.A.; TRINDADE, R.I.F.; GOGUITCHAICHVILI, A.; AFONSO, M.C.

2007a Archaeointensity from 1300-200 BP Brazilian Pottery. In: 2007 Joint Assembly, American Geophysical Union.

HARTMANN, G.A.; AFONSO, M.C.; TRINDADE, R.I.F.

2007b Arqueomagnetismo no Brasil: primeiros resultados de cerâmicas históricas e préhistóricas. In: 1० Simpósio Latino Americano sobre Métodos Físicos e Químicos em Arqueologia, Arte e Conservação de Patrimônio Cultural, Sociedade Brasileira de Física. MASP, São Paulo, junho de 2007: 19.

KIRSCHVINK, J.L.

1980 The least-squares line and plane and analysis of paleomagnetic data. Geophys. J. R. Astron. Soc., 62: 699-718.

KORTE, M.; GENEVEY, A.; CONSTABLE, C.G.; FRANDK, U.; SCHNEPP E.

2005 Continuous geomagnetic field models for the past 7 millennia: 1 . A new global data compilation, Geochem. Geophys. Geosyst., 6: Q02H15.

KOVACHEVA, M.

1997 Archaeomagnetic database from Bulgaria: the last 8000 years. Phys. Earth Planet. Int., 102: 145-151.

KOVACHEVA, M.; HEDLEY, I.; JORDANOVA, N.; KOSTADINOVA, M.; GIGOV, V.

2004 Archaeomagnetic dating of archaeological sites from Switzerland and Bulgaria. J. Archaeol. Sci., 31: 1463-1479.

KOVACHEVA, M; JORDANOVA, N.; KARLOUKOVSKI, V.

1998 Geomagnetic field variations as determined from Bulgarian archaeomagnetic data. Part II: the last 8000 years. Surv. Geophys., 19: 431-460.

LABELLE, J.M.; EIGMY, J.L.

1997 Additional archaeomagnetic data on the variations as determined from Bulgarian archaeomagnetic data. Part II: the last 8000 years. Surv. Geophys., 9(5): 413-439.

LANOS, PH.; LE GOFF, M.; KOVACHEVA, M; SCHNEPP, E.

2005 Hierarchical modelling of archaeomagnetic data and curve estimating by moving average technique. Geophys. J. Int., 160: 440-476.

LE GOFF, M.; GALlet, Y.; GENEVEY, A.; WARME, N.

2002 On archaeomagnetic secular variation 
curves and archaeomagnetic dating. Phys. Earth Planet. Int., 134, (3-4): 203-211.

MCFADDEN, P.L.; MCELHINNY, M.W.

1990 Classification of the reversal test in palaeomagnetism. Geophys. J. Int., 103: 725-729.

MCINTOSH, G.; CATANZARITI, G.

2006 An introduction to archaeomagnetic dating, Geochronometria, 25: 11-18.

MERRILL, R.T.; MCELHINNY, M.W.; MCFADDEN, P.L.

1998 The Magnetic Field of the Earth: Paleomagnetism, the Core, and the Deep Mantle. International Geophysics Series, vol. 63, Academic Press.

MORALES, J.; GOGUITCHAICHVILI, A.; URRUTIA-FUCUGAUCHI, J.

2001 A rock-magnetic and paleointensity study of some Mexican volcanic lava flows during the Latest Pleistocene to the Holocene. Earth Planets Space, 53: 893-902.

NAGATA, T.; ARAI, Y.; MOMOSE, K.

1963 Secular variation of the geomagnetic total force during the last 5000 years. J. Geophys. Res., 68: 5277-5281.

PARÉS, J.M.; PÉREZ-GONZÁLEZ, A.; ROSAS, A.; BENITO, A.; BERMÚDEZ DE CASTRO, J.M.; CARBONELL, E.; HUGUET, R.

2006 Matuyama-age lithic tools from the Sima del Elefante site, Atapuerca (northern Spain). J. Hum. Evol., 50: 163-169.

PERRIN, M.; SCHNEPP, E.

2004 IAGA paleointensity database: distribution and quality of the data set. Phys. Earth Planet. Int., 147: 255-267.

PERRIN, M.; SCHNEPP, E.

2004 IAGA paleointensity database: distribution and quality of the data set. Phys. Earth Planet. Int., 147: 255-267.

SCHNEPP, E; LANOS, PH.

2005 Archaeomagnetic secular variation in
Germany during the past 2500 years. Geophys. J. Int., 163: 479-490.

SOLER-ARECHALDE, A.M.; SANCHEZ, F.; RODRIGUEZ, M.; GOGUITCHAICHVILI, A.; URRUTIA-FUCUGAUCHI, J.

2006 Archaeomagnetic investigation of oriented pre-Columbian lime-plasters from Teotihuacan, Mesoamerica. Earth Planets Space, 58: 1433-1439, 2006.

STENBERG, R.

1999 Secular variation of archaeomagnetic direction in the American southwest, AD 750-1452. J. Geophys. Res., 94 (B1): $527-546$

TANGUY, J-C.; LE GOFF, M.; PRINCIPE, C.; ARRIGHI, S.; CHILLEMI, V.; PAIOTTI, A.; LA DELFA, S.; PATÀNE, G.

2003 Archaeomagnetic dating of Mediterranean volcanics of the last 2100 years: validity and limits. Earth Planet. Sci. Lett., 211: 111-124.

TAUXE, L.

2005 Lectures in Paleomagnetism http:// earthref.org/MAGIC/books/Tauxe/ 2005.

THELLIER, E.; THELLIER, O.

1959 Sur l'intensité du champ magnetique terrestre dans le passé historique et géologique. Ann. Geophys., 15: 285-376.

VALET, J.-P.

2003 Time Variations in Geomagnetic Intensity. Rev. Geophys., 41: 1.

YU, Y.; DUNLOP, D.J.; PAVLISH, L.; COOPER, M.

2000 Archaeomagnetism of Ontario potsherds from the last 2000 years. J. Geophys. Res., 105(B8): 19419-19434.

ZANELLA, E.; GURIOLI, L.; CHIARI, G.; CIARALLO, A.; CIONI, R.; DE CAROLIS, E.; LANZA, R.

2000 Archaeomagnetic results from mural paintings and pyroclastic rocks in Pompeii and Herculaneum. Phys. Earth Planet. Int., 118: 227-240. 